\title{
Effects of Maternal Vitamin D Supplementation on the Maternal and Infant Epigenome
}

\author{
Cindy M. Anderson,,"* Shannon L. Gillespie,,"* Doria K. Thiele, \\ Jody L. Ralph,,3 and Joyce E. Ohm ${ }^{4}$
}

\begin{abstract}
Introduction: Mothers and infants are at high risk for inadequate vitamin D status. Mechanisms by which vitamin D may affect maternal and infant DNA methylation are poorly understood.

Objective: This study quantified the effects of vitamin $\mathrm{D}_{3}$ supplementation on DNA methylation in pregnant and lactating women and their breastfed infants.

Materials and Methods: In this randomized controlled pilot study, pregnant women received vitamin $\mathrm{D}_{3} 400$ international units (IU) ( $n=6$; control) or 3,800 IU ( $n=7$; intervention) daily from late second trimester through 4-6 weeks postpartum. Epigenome-wide DNA methylation was quantified in leukocytes collected from mothers at birth and mother-infant dyads at 4-6 weeks postpartum.

Results: At birth, intervention group mothers showed DNA methylation gain and loss at 76 and 89 cytosineguanine $(\mathrm{CpG})$ dinucleotides, respectively, compared to controls. Postpartum, methylation gain was noted at 200 and loss at 102 CpGs. Associated gene clusters showed strongest biologic relevance for cell migration/ motility and cellular membrane function at birth and cadherin signaling and immune function at postpartum. Breastfed 4-6-week-old infants of intervention mothers showed DNA methylation gain and loss in 217 and 213 CpGs, respectively, compared to controls. Genes showing differential methylation mapped most strongly to collagen metabolic processes and regulation of apoptosis.

Conclusions: Maternal vitamin D supplementation during pregnancy and lactation alters DNA methylation in mothers and breastfed infants. Additional work is needed to fully elucidate the short- and long-term biologic effects of vitamin D supplementation at varying doses, which could hold important implications for establishing clinical recommendations for prenatal and offspring health promotion.
\end{abstract}

Keywords: DNA methylation, epigenomics, pregnancy, breastfeeding, infant, lactation

\section{Introduction}

$\mathbf{V}$ ITAMIN D IS A FAT-SOLUBLE prohormone obtained through exposure to sunlight or orally in the form of few natural foods, some fortified foods, or supplements, with serum status subject to genetic and additional environmental influences. ${ }^{1}$ The Institute of Medicine (IOM) recommends that pregnant and lactating women receive 600 international units (IU) daily and infants receive 400 IU daily of vitamin D and defines a 25-hydroxyvitamin $\mathrm{D}[25(\mathrm{OH}) \mathrm{D}]$ blood level of $<20 \mathrm{ng} / \mathrm{mL}$ as deficiency. ${ }^{2}$ Others (e.g., the Endocrine Society, ${ }^{3}$ Holick $^{4}$ ) propose that significantly greater intake may be required among mothers and infants to achieve sufficiency [i.e., $25(\mathrm{OH}) \mathrm{D}$ levels $\geq 30 \mathrm{ng} / \mathrm{mL}$ ] and to achieve optimal benefits [i.e., $25(\mathrm{OH}) \mathrm{D}$ levels $\geq 40 \mathrm{ng} / \mathrm{mL}$ ]. A large number of women and infants fail to meet even the IOM recommendations, resulting in U.S. rates of vitamin D deficiency ranging from $14 \%$ to $72 \%$, dependent upon the location and characteristics of the sample under study. ${ }^{5,6}$ During pregnancy and lactation, maternal vitamin D plays a well-established role in calcium homeostasis during fetal osteogenesis and infant bone mineralization. More recently, lower maternal vitamin D status has been witnessed in the context of gestational diabetes, ${ }^{7}$ preterm birth, ${ }^{8}$ preeclampsia, ${ }^{9}$ postpartum weight retention, ${ }^{10}$ and infant respiratory tract infection. ${ }^{11}$ Maternal vitamin D deficiency has also been linked to long-term offspring illness,

\footnotetext{
${ }^{1}$ Center for Women, Children and Youth, The Ohio State University College of Nursing, Columbus, Ohio.

${ }^{2}$ Department of Nursing, University of North Dakota College of Nursing and Professional Disciplines, Grand Forks, North Dakota.

${ }^{3}$ University of Windsor, Faculty of Nursing, Windsor, Ontario, Canada.

${ }^{4}$ Department of Cancer Genetics and Genomics, Roswell Park Cancer Institute, Buffalo, New York.

*These authors contributed equally to the writing of this article.
} 
such as risk for type 1 diabetes ${ }^{12}$ and eczema. ${ }^{13}$ These findings suggest that vitamin D plays a larger role in maintaining maternal and offspring health than once appreciated, although mechanisms have not been elucidated.

The short-term effects of maternal vitamin D deficiency on infant health may be explained by associations among maternal and infant vitamin D status due to placental and breast milk transfer. ${ }^{14}$ Given the time lapse between pregnancy, lactation, and more long-term health outcomes of the child, the potential for fetal programming as mediated by epigenomic effects of maternal vitamin D status has also been of interest. Specifically, addition or removal of methyl groups to phosphate-linked cytosine-guanine $(\mathrm{CpG})$ dinucleotides (i.e., DNA methylation) has been implicated as a potential mechanism linking in utero and early life vitamin D status to long-term offspring gene expression (reviewed by Wright et al. ${ }^{15}$ ).

Vitamin D status has been shown to affect the epigenome of nonpregnant/nonlactating adults, which may help to explain associations among maternal vitamin D deficiency and complications of pregnancy. ${ }^{16}$ Moreover, animal models now link maternal vitamin D status to the methylation and expression of several targeted genes. ${ }^{17,18}$ Epigenome-wide comparison of offspring born to vitamin D supplemented versus depleted pregnant Sprague-Dawley rats has also identified differential DNA methylation with downstream implications for offspring health. ${ }^{19}$

Studies of this nature have been rare in humans, with null results noted upon examination of associations among circulating maternal $25(\mathrm{OH}) \mathrm{D}$ levels and targeted ${ }^{20}$ or epigenome-wide DNA methylation in infant cord blood. ${ }^{21}$ The effects of maternal vitamin D supplementation, allocated randomly, on maternal and infant DNA methylation across the epigenome remain to be determined, but have the potential to generate novel evidence for the molecular underpinnings of vitamin D-related health outcomes. Therefore, this study aimed to identify the combined effect of pre- and postnatal maternal vitamin D supplementation on epigenomewide DNA methylation of leukocytes sampled from women at birth, these same women after 4-6 weeks of lactation, and their breastfed infants at 4-6 weeks of life.

\section{Materials and Methods}

\section{Design}

This study used a double-blind, randomized controlled design as previously reported in detail. ${ }^{14}$ Briefly, following approval by the institutional review board, study recruitment and enrollment commenced. Potential participants were approached by research team members and provided with written and verbal descriptions of the study and participant requirements. All study participants provided written informed consent and HIPAA authorization before data collection.

\section{Setting}

Women were recruited from a single Midwestern hospitalbased obstetric practice when they presented for routinely scheduled prenatal care between 24 and 28 weeks of gestation. Data collection occurred from July 2012 through January 2013.

\section{Sample}

Sampling was by convenience. Women 18 years or older, with a current pregnancy between 24 and 28 weeks, intent to breastfeed for at least 4-6 weeks, and history of breastfeeding for at least 4 weeks with a prior infant (to mitigate attrition) met inclusion criteria. Exclusion criteria included preexisting type 1 or type 2 diabetes, hypertension, parathyroid disease, uncontrolled thyroid disease, and use of vitamin D supplements beyond a prenatal vitamin in the last 6 months.

Participants were randomized to one of two study groups through allocation concealment based on an a priori sequence. Blinding of the intervention was maintained at the level of participants, the data collectors, and the data analyst until completion of all data collection. Participants randomized to the placebo arm received prenatal vitamins containing 400 IU vitamin $\mathrm{D}_{3}$ plus a placebo capsule (both taken daily). Participants randomized to the intervention arm received prenatal vitamins containing $400 \mathrm{IU}$ vitamin $\mathrm{D}_{3}$ plus a vitamin D capsule containing 3,400 IU (both taken daily). Participants were instructed to take their assigned supplements daily starting on the day of enrollment through 4-6 weeks postpartum and were contacted bimonthly for evaluation of intervention fidelity and to maintain participant interest. All participants were also encouraged to supplement their infants at $400 \mathrm{IU} /$ daily vitamin $\mathrm{D}$ according to recommendations for breastfed infants. $^{2}$

\section{Measurement}

Dietary intake. The Food Frequency Questionnaire $\left(\mathrm{FFQ}^{22}\right.$ ) was administered to women at enrollment and at the postpartum visit to assess their dietary intake over the prior 3 months. The FFQ is akin to the Harvard Service FFQ ${ }^{23}$ and allows for the quantification of self-reported dietary intake, including calcium and vitamin D. Infant dietary intake from nonbreast milk sources was recorded using an investigatorgenerated instrument. Infants receiving breast milk with no other liquids or solids with the exception of recommended vitamin D 400 IU supplementation were categorized as exclusively breastfed. ${ }^{24}$ Infants receiving breast milk and other liquids or solids were categorized as partially breastfed (high, $>80 \%$; medium, 20-80\%; low $<20 \%$ ) based on relative quantity of breast milk consumed. ${ }^{25}$

Serum analytes. Maternal and infant serum were analyzed for 25-hydroxyvitamin D [25(OH)D], a metabolite of vitamin $\mathrm{D}$ that serves as the principle circulating reservoir and indicator of overall vitamin D status, by enzyme immunoassay (Immunodiagnostic Systems Ltd., Gaithersburg, MD). Measures of maternal serum calcium (BioVision Calcium Colorimetric Assay) and parathyroid hormone (PTH) by enzyme-linked immunoassay (Abnova, Walnut, CA) were also completed at each data collection point.

DNA methylation. DNA was extracted from maternal and infant leukocytes as previously described in detail. ${ }^{26,27} \mathrm{Me}$ thylation from purified genomic DNA $(3 \mu \mathrm{g})$ was quantified using the Illumina Infinium DNA methylation $450 \mathrm{~K}$ bead-based array. ${ }^{28-30}$ Biological relevance of differentially methylated genes was determined using the Database for 
Annotation, Visualization and Integrated Discovery (DAVID) v6.8..$^{31,32}$

\section{Data collection}

Women were enrolled at 24-28 weeks of pregnancy. At this time, self-reported maternal demographic characteristics were recorded. At enrollment and at 4-6 weeks postpartum, participants completed the FFQ. Infant supplemental vitamin D intake was recorded by maternal report for the 4-6 week period. Venipuncture was performed in women at enrollment, birth, and at 4-6 weeks postpartum, with serum samples at each time point serving as the source for $25(\mathrm{OH}) \mathrm{D}$, calcium, and PTH quantification and isolated leukocytes at birth and 4-6 weeks postpartum serving as the source for DNA methylation analyses. Infant heel stick was also performed at 4-6 weeks of age. Leukocytes were isolated and genomic DNA extracted and purified for use in infant DNA methylation analysis. Data were also obtained through maternal medical record abstraction at enrollment and at 4-6 weeks postpartum, including prepregnancy weight, current weight, height, gravidity, parity, history of significant medical conditions, date of last menstrual period, obstetric estimate of due date, characteristics of labor and birth, and newborn anthropometric measurements.

\section{Data analysis}

Maternal characteristics and serum analytes were examined and compared among the control versus intervention group. Differentially methylated $\mathrm{CpG}$ sites were identified using the GenomeStudio DNA methylation module (Illumina) following normalization of methylation data. Average beta scores for each individual $\mathrm{CpG}$ site ranging from $0(0 \%$ methylation) to 1 (100\% methylation) within a given sample were compiled for mothers and infants in the intervention and control groups. Individual $\mathrm{CpG}$ dinucleotides having a change in beta (delta.beta) score greater than 0.2 (indicating $>20 \%$ difference in methylation compared to controls) and a $p$-value $<0.05$ by two-tailed $t$ test were defined as having significantly differential methylation. Intention to treat guided serum measures of $25(\mathrm{OH}) \mathrm{D}$, calcium, and PTH with significance determined by two-tailed $t$ test $(p$-value $<0.05)$. Biological relevance of differentially methylated genes was determined through functional annotation clustering using DAVID bioinformatics resources based on gene ontology and enrichment score (geometric mean of all enrichment $p$-values for each annotation term associated with gene members in the group) to determine rank importance of biological functions for associated genes. ${ }^{32}$ Functional annotation clustering explores group by group annotations, rather than one by one singular terms, to identify related genes in a network format (false discovery rate $<0.05$ for multiple testing correction). Enrichment scores enable interpretation of biologic relevance of functionally related genes and their relationship in a network format, ranking overall importance of association with gene members in a group. Scores that indicate importance of gene members are typically higher, with enrichment scores consistent with importance. Enrichment scores $>1.3$ reflect greater importance although lower scores retain biological plausibility.

\section{Results}

\section{Participant characteristics}

As shown in Figure 1, among the 16 participants randomized to the control and intervention groups ( $n=8$ /group), 13 participated in the study (control group, $n=6$; intervention group, $\left.n=7^{14}\right)$. Data were available for all participants at birth but, due to loss to follow-up, analysis was limited to three control group dyads and seven intervention group dyads at the 4-6 week postpartum time point. As previously reported, ${ }^{14}$ all participants reported white race, and the control and intervention groups did not significantly differ in maternal age, parity, body mass index, dietary vitamin D intake, or dietary calcium intake at enrollment ( $p$-values $>0.21)$. As reported, ${ }^{14}$ timing of randomization and timing of birth did not significantly differ among the groups ( $p$-values $\geq 0.22$ ), allowing for $\sim 11$ weeks of prenatal vitamin $\mathrm{D}$ supplementation or placebo before sampling at birth and an additional 4-6 weeks of postnatal supplementation or placebo before sampling at postpartum. As shown in Table 1, vitamin D supplementation among the intervention group significantly increased serum $25(\mathrm{OH}) \mathrm{D}$ levels among both mothers and infants while maintaining appropriate maternal calcium and PTH serum concentrations, indicating efficacy and safety of the intervention. Infant vitamin D supplementation was highly variable, with $1 / 3$ infants in the control group and 5/7 infants in the intervention group receiving no vitamin $D$ supplementation during the follow-up period per maternal report $(X=1,067 \mathrm{IU}, \mathrm{SD}=1,514 \mathrm{IU} ; X=1,714 \mathrm{IU}, \mathrm{SD}=3,462$ total dose among control and intervention infants, respectively).

\section{Epigenome-wide comparisons of DNA methylation}

First, we identified CpGs showing significantly different maternal leukocyte DNA methylation among the control versus intervention mothers at birth and at 4-6 weeks postpartum. As evidenced by a delta.beta of $\geq 0.2$ and $p$-value of $\leq 0.05$ per two-tailed comparison, adequate vitamin D supplementation was associated with significant maternal DNA methylation gain at 76 and loss at 89 CpGs at birth (Fig. 2a) and gain at 200 and loss at 102 CpGs at postpartum (Fig. 2b) among the intervention versus control group. Next, we compared epigenome-wide leukocyte DNA methylation at 4-6 weeks of life among intervention versus control infants. Per maternal self-report, all control infants with available data were exclusively breastfed $(n=3)$. Among infants in the intervention group, three were exclusively breastfed, and the remaining four were categorized as partially breastfed (high), consuming a low relative quantity of formula (mean 306.0 [SD 516.3] total $\mathrm{mL} ; 10.9 \mathrm{~mL}$ daily) from birth to time of assessment. $^{24,25}$ Among infants in the intervention group, 217 CpGs were identified with significant methylation gain and 213 CpGs with significant methylation loss (Fig. 2c).

\section{Biological relevance of differential DNA methylation}

Next, we examined the biological relevance of differential DNA methylation by functionally annotating gene sets associated with differentially methylated $\mathrm{CpG}$ dinucleotides using DAVID bioinformatics resources. Among mothers, CpGs with significant methylation gain among the intervention versus control group were associated with 45 known genes at birth and 130 known genes at postpartum. Twenty-four 


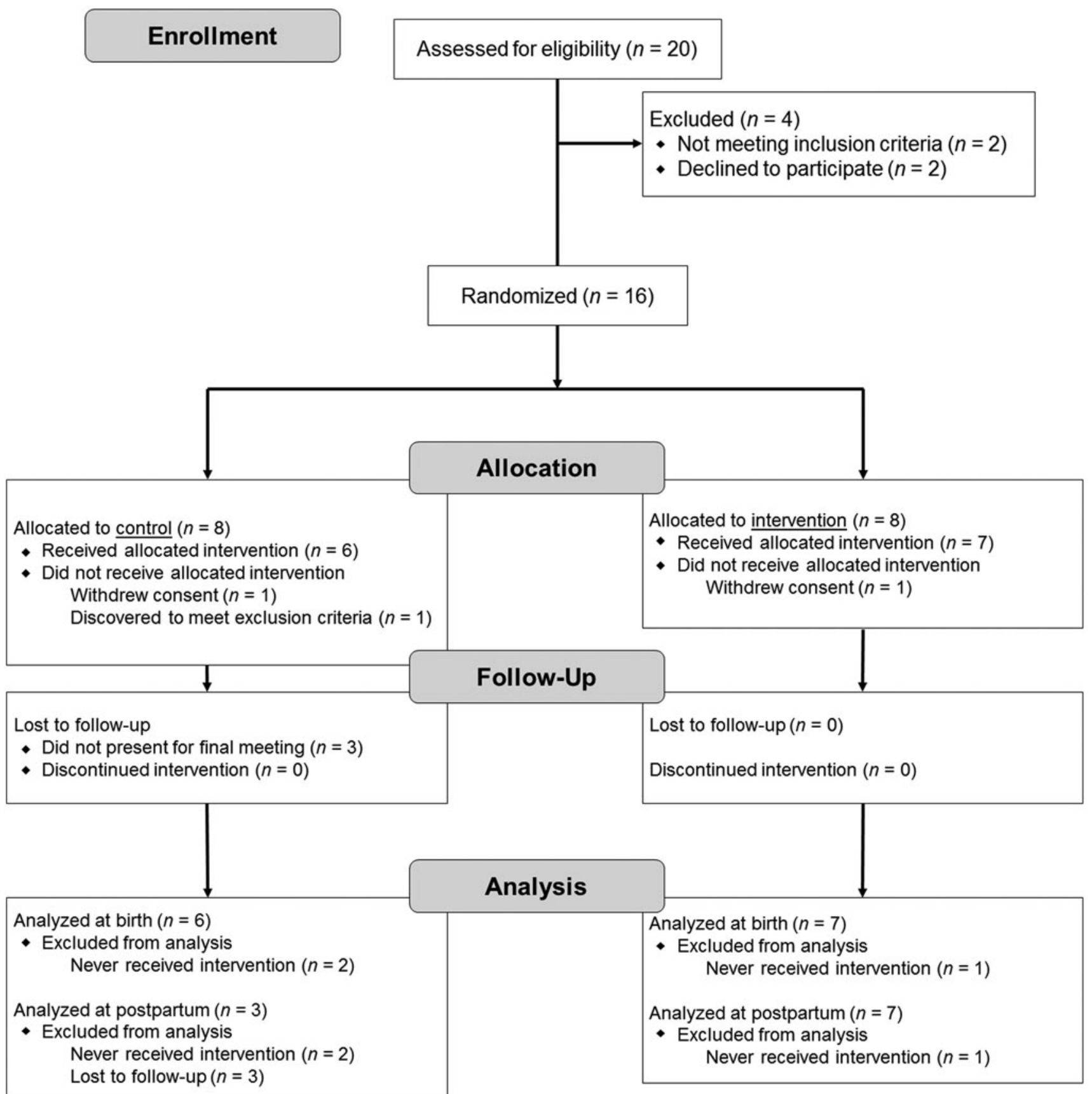

FIG. 1. CONSORT diagram for enrollment, allocation, follow-up, and analysis. Reproduced in edited form with permission from Thiele et al. ${ }^{14}$

of these genes were common to both sampling time points. Functional annotation clustering results are presented in Table 2, with genes associated with $\mathrm{CpGs}$ with methylation gain showing the strongest functional relevance for cell migration/motility at birth (enrichment score $=2.7$ ) and cadherin signaling at postpartum (enrichment score $=18.23$ ). Focusing on the 24 genes with gain in methylation common to both the birth and postpartum assessments, relevant biological associations included metabolic processes, transcription, ion binding, and cellular membrane function (data not shown), suggesting that these processes are significantly more enriched or important in this study.
Maternal leukocyte $\mathrm{CpGs}$ with significant loss among the intervention versus control group were associated with 52 known genes at birth and 70 known genes at postpartum, with 23 genes showing overlap between the two time points. The functional clusters associated with methylation loss are also presented in Table 2. While no cluster exceeded an enrichment score of 1.3 at birth, which indicates greater importance, it is notable that differential regulation of cellular membrane processes and ion binding would be expected to show functional implications for bone and calcium homeostasis, as well as a wide range of cellular processes. During postpartum, genes associated with methylation loss most strongly mapped 
Table 1. Serum Markers of Maternal and Infant Vitamin D Status and Maternal Regulation

\begin{tabular}{lcc}
\hline Analyte & $\begin{array}{c}\text { Control } \\
\text { group }\end{array}$ & $\begin{array}{c}\text { Intervention } \\
\text { group }\end{array}$ \\
\hline Maternal & & \\
25(OH)D (ng/mL) & $32.4(3.5)$ & $31.4(4.3)$ \\
$\quad$ Baseline & $23.7(2.9)$ & $33.1(2.7)^{*}$ \\
$\quad$ Birth & $21.9(2.5)$ & $36.0(2.3)^{*}$ \\
4-6 weeks postpartum & & \\
Calcium (mg/dL) & $8.7(0.2)$ & $8.8(0.4)$ \\
Baseline & $8.4(0.2)$ & $8.5(0.4)$ \\
Birth & $9.2(0.3)$ & $9.2(0.3)$ \\
4-6 weeks postpartum & & \\
PTH (pg/mL) & $45.1(18.1)$ & $42.5(14.8)$ \\
Baseline & $33.5(17.3)$ & $36.0(19.3)$ \\
Birth & $47.1(28.9)$ & $60.6(20.1)$ \\
4-6 weeks postpartum & & \\
Infant & & \\
25(OH)D (ng/mL) & $23.7(5.5)$ & $32.3(6.1)^{*}$ \\
Birth & $11.4(8.4)$ & $24.9(12.8)^{* *}$ \\
4-6 weeks of life &
\end{tabular}

Values at baseline are based on 8 participants per group. Values at birth are based on 6 control group participants and 7 intervention group participants. Values at 4-6 weeks postpartum are based on 3 control group mother-infant dyads and 7 intervention group mother-infant dyads.

$* p<0.05$ and $* * p<0.10$ for control and intervention group comparisons by two-sided $t$ tests.

25(OH)D, 25-hydroxyvitamin D; PTH, parathyroid hormone.

to the immune function cluster (enrichment score $=1.36$ ). Also of interest, functional clusters of genes regulating transcription and hormone regulation arose during the postpartum assessment but were not present at birth. In analyzing the 23 genes linked to methylation loss at both birth and postpartum, relevant biological associations included cellular membrane, DNA-dependent transcription, cation binding, and glycosylation (data not shown).

Among leukocytes sampled from infants at 4-6 weeks of life, $\mathrm{CpG}$ dinucleotides showing methylation gain following maternal pre- and postnatal vitamin D supplementation were associated with 129 known genes, and $\mathrm{CpG}$ dinucleotides showing methylation loss were associated with 135 known genes. Functional clustering of differentially methylated gene sets is shown in Table 2. In this study, collagen metabolic processes (enrichment score $=1.82$ ) and regulation of apoptosis (enrichment score $=1.61$ ) showed the highest enrichment scores for genes linked to $\mathrm{CpG}$ loci with gain and loss of methylation, respectively.

\section{Discussion}

Health outcomes due to lower vitamin D status often do not present until there is evidence of severe disease, underscoring the importance of identification of mechanistic underpinnings for deficiency or insufficiency across the life span. As vitamin $\mathrm{D}$ is known to regulate DNA methylation, we sought to identify the combined effect of pre- and postnatal vitamin D supplementation on leukocyte epigenome-wide DNA methylation in pregnant and lactating women and their breastfed infants as a first step in unraveling the molecular underpinnings linking vitamin D status with related health outcomes during critical developmental stages.

Epigenetic investigation linking vitamin D status with health outcomes has been conducted in cell culture, animal, and human studies. ${ }^{17-20,33} \mathrm{We}$, and others, have investigated vitamin D-associated DNA methylation in mothers during a single time point in pregnancy ${ }^{20,21,26}$ and in placental tissue. ${ }^{2,34}$ In this study, we extend observations of vitamin D-associated differences in DNA methylation in women at birth and during early lactation and among their breastfed infants. Our findings provide evidence of maternal and infant vitamin D status as a potential driver of differential DNA methylation at multiple loci across the maternal and infant epigenomes, providing initial evidence of putative molecular targets for long-term health outcomes.

In this study, maternal supplementation with 3,800 IU of vitamin $\mathrm{D}$ per day was associated with mean $25(\mathrm{OH}) \mathrm{D}$ levels reflective of sufficient maternal vitamin $\mathrm{D}$ status by birth among the intervention group, as well as significant gains in maternal leukocyte DNA methylation associated with genes involved in cell migration/motility, development, and growth. The implications of methylation gain for gene expression and pathway activity remain to be determined. It is well known that pathways regulating blood vessel development are central to biological processes associated with the demands for a hypertrophic maternal circulatory system, and continued development of the circulatory needs of the placenta and maternal vitamin D status has been shown to affect placental vascular endothelial growth factor gene expression. ${ }^{35}$ Sufficient maternal vitamin D levels in pregnancy among the intervention group were also associated with methylation loss at birth in pathways enriched for genes regulating metabolic, immune, and neurologic function, providing further insight to potential mechanisms underlying adverse outcomes in pregnancy, including gestational diabetes, preterm birth, and preeclampsia. Consistent with findings that deficient maternal vitamin D status in pregnancy may have adverse effects on motor and social development in children, ${ }^{36}$ differential DNA methylation regulating

FIG. 2. Heat map showing cytosine-guanine $(\mathrm{CpG})$ dinucleotides with differential methylation. Leukocyte $\mathrm{CpG}$ dinucleotides with a significant gain or loss of methylation (delta.beta $>0.2$ or $<-0.2$, respectively; $p<0.05$ ) among the intervention versus control group, with women in the intervention group receiving daily prenatal vitamins containing 400 international units (IU) vitamin $\mathrm{D}_{3}$ plus a vitamin D capsule containing 3,400 IU and women in the control group receiving daily prenatal vitamins containing $400 \mathrm{IU}$ vitamin $\mathrm{D}_{3}$ plus a placebo capsule. Data displayed compare DNA methylation quantified using the Illumina Infinium $450 \mathrm{~K}$ array, with genomic DNA templates isolated from: (a) maternal leukocytes at time of birth, (b) maternal leukocytes at 4-6 weeks postpartum, and (c) infant leukocytes at 4-6 weeks of life. For each heat map, samples are displayed on the horizontal axis (control group: green, intervention group: orange), while differentially methylated $\mathrm{CpG}$ is displayed on the vertical axis. Methylation is displayed as a beta score ranging from 0 (unmethylated: blue) to 1 (fully methylated: red). $\mathrm{CpG}$ is classified based on their relation to cataloged $\mathrm{CpG}$ islands with a colored bar on the vertical axis (CpG island: red, shelf regions: light blue, shore regions: purple, open sea: dark blue). 
a

Color Key 今。

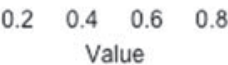

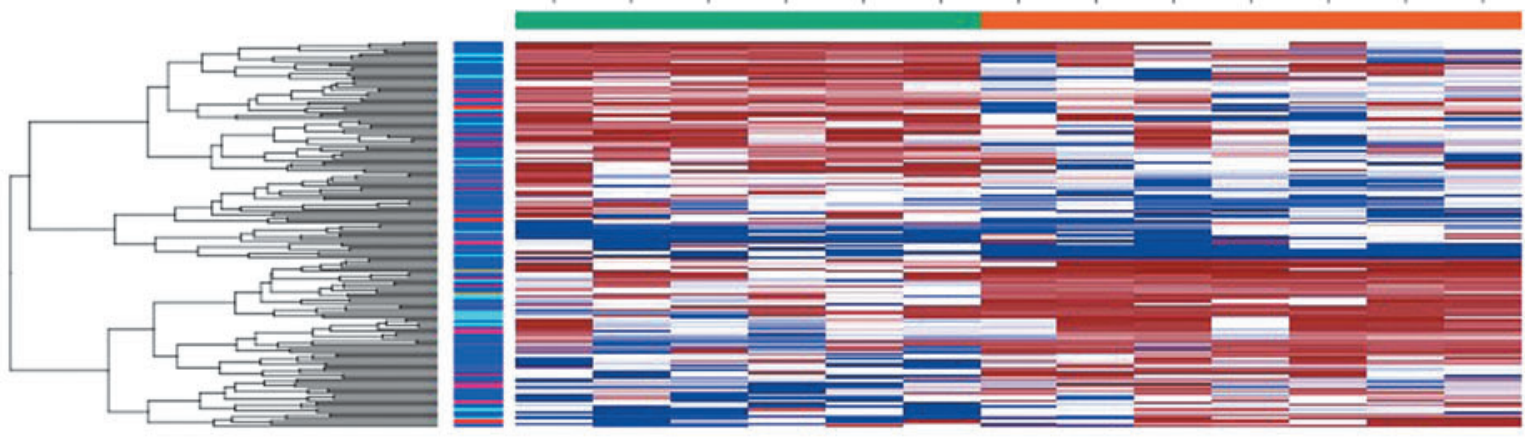

$\square$ Island $\square$ Shelf $\quad$ Shore $\quad$ Open Sea $\square$ Control $\square$ Intervention
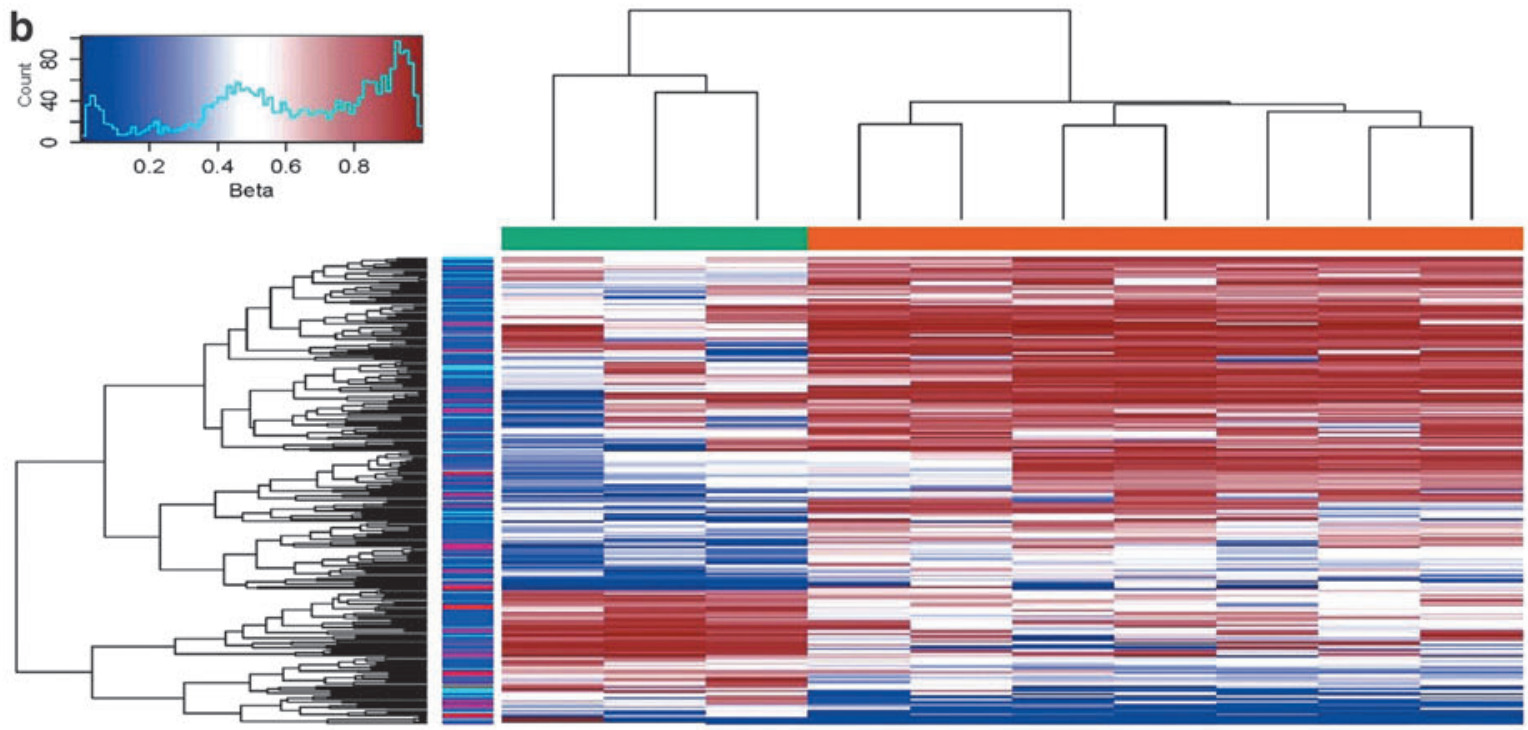

C
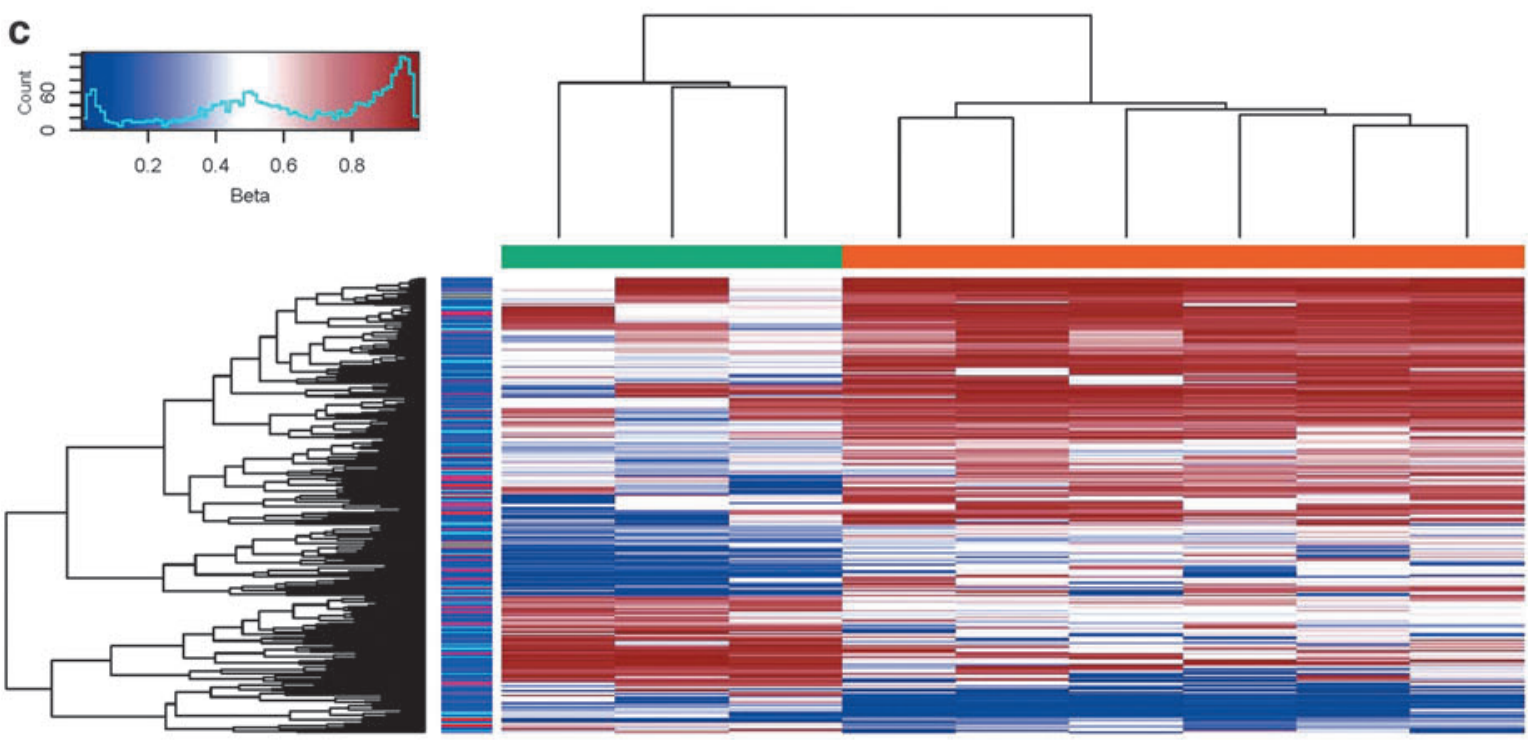
Table 2. Biological Relevance of Genes Associated with Differentially Methylated Cytosine-Guanine Dinucleotides

\begin{tabular}{|c|c|c|c|}
\hline & Functional cluster & $\begin{array}{l}\text { Enrichment } \\
\text { score }\end{array}$ & Associated genes \\
\hline \multicolumn{4}{|c|}{ Maternal methylation gain } \\
\hline \multirow[t]{6}{*}{ At birth } & Cell migration/motility & 2.7 & $G A B 2, L M X 1 B, A N G, C D H 13, T G F B R 3$ \\
\hline & Regulation of cell development/size & 1.81 & CDH4, HDAC4, MAPT, TGFBR3 \\
\hline & Blood vessel development & 1.57 & ARHGAP22, ANG, CDH13, TGFBR3 \\
\hline & Regulation of gene expression & 1.39 & LMX1B, PRDM16, CDH13, HDAC4, LIF, RSF1 \\
\hline & Regulation of cell growth & 1.09 & CDH13, CDH4, MAPT \\
\hline & Regulation of transcription & 1.0 & $\begin{array}{l}\text { LMX1B, PRDM16, ARHGAP22, HDAC4, LIF, } \\
\quad \text { RSF1, TGFBR3, ZNF135, ZNF707 }\end{array}$ \\
\hline \multirow{7}{*}{$\begin{array}{c}\text { At } 4-6 \text { weeks } \\
\text { postpartum }\end{array}$} & Cadherin signaling & 18.23 & PCDHGA1-11, PCDHGB1-7 \\
\hline & Small GTPase signal transduction & 2.65 & $\begin{array}{l}\text { MCF2, ARHGEF10, KNDC1, PREX1, PSD3, } \\
\quad \text { VAV2 }\end{array}$ \\
\hline & Ion binding & 2.17 & $\begin{array}{l}\text { ATP8B3, MUS81, MGMT, PRDM16, PDCD6, } \\
\text { ANG, CLSTN2, C11orf54, MAST1, NOS2, } \\
\text { KCNQ2, PCDHGA1-11, PCDHGB1-7, RSF1, } \\
\text { SCGN, SFXN1, SLC23A2, SORBS2, SRI, SDF4, } \\
\text { VAV2, ZNF135, ZNF707 }\end{array}$ \\
\hline & Calcium binding & 1.42 & PDCD6, SCGN, SRI, SDF4 \\
\hline & Regulation of developmental growth & 1.26 & $H O P X, M A P T, N R G 1$ \\
\hline & Nuclease activity & 1.11 & MUS81, ANG, RNASE4 \\
\hline & Regulation of transcription & 1.01 & PRDM16, BRDT, MYCBP, DCC, MAML3, NRG1 \\
\hline \multicolumn{4}{|c|}{ Maternal methylation loss } \\
\hline \multirow[t]{5}{*}{ At birth } & Cellular membrane & 0.75 & $\begin{array}{l}\text { GRP133, GREB1, ASAH2, RASA3, ASAM, AIG1, } \\
\text { MF12, C7orf45, IGSF3, IL12RB1, LHFP, } \\
\text { NINJ2, OR12D3, OR2 L13, PARD3B, PTGDR, } \\
\text { PTPRN2, RFTN1, SCL29A4, TMC3 }\end{array}$ \\
\hline & Metabolic processes & 0.67 & DNAJA3, GDF7, MAPK8IP3 \\
\hline & Immune function & 0.63 & $A S A M, A C A N, I G S F 3$ \\
\hline & $\begin{array}{l}\text { Neurologic, cognition, } \\
\text { sensory perception }\end{array}$ & 0.24 & POU6F2, OR12D3, OR2 L13 \\
\hline & Ion binding & 0.14 & $\begin{array}{l}\text { DNAJA3, RASA3, MF12, ADAMTSL2, ZNF879, } \\
\quad \text { TRPS1, ZC3H12D }\end{array}$ \\
\hline \multirow[t]{6}{*}{$\begin{array}{c}\text { At } 4-6 \text { weeks } \\
\text { postpartum }\end{array}$} & Immune function & 1.36 & $\begin{array}{l}\text { HEPACAM2, IGSF3, LING01, HLA-A, } \\
\quad \text { HLA-DRB1, MOG }\end{array}$ \\
\hline & Cellular membrane & 0.93 & $\begin{array}{l}\text { ATP2C2, HEPACAM2, KIAA1467, RASA3, } \\
\text { C7orf45, CACNA1C, IGSF3, IL12RB1, LING01, } \\
\text { HLA-A, HLA-DRB1, MOG, NINJ2, PLXNC1, } \\
\text { PTGDR, SLC2A9, SLC25A21, SLC26A1, } \\
\text { SLC9A9, TMC3, UMODL1 }\end{array}$ \\
\hline & Transcription negative regulation & 0.81 & NFIC, PIAS4, SIRT5, TRPS1 \\
\hline & Transcription DNA dependent & 0.65 & POU6F2, NFIC, TRPS1 \\
\hline & Ion transport & 0.56 & ATP2C2, CACNA1C, SLC6A1, SLC9A3, SLC9A9 \\
\hline & Hormone stimulus response & 0.35 & CCNA2, PRKAR1B, SLC9A3 \\
\hline \multicolumn{4}{|c|}{ Infant methylation gain } \\
\hline At $4-6$ weeks & Collagen metabolic processes & 1.82 & ADMTS2, MMP27, TNXB \\
\hline of life & Lung development & 1.44 & ADMTS2, MGP, PDGFRA, TGFB3 \\
\hline & Ossification & 1.35 & GNAS, GABBR1, MGP, TGFB3 \\
\hline & Palate development & 1.04 & PDGFRA, TFGB3, TGFRB3 \\
\hline & In-utero embryonic development & 0.94 & PDGFRA, TFGB3, TGFRB3, ZMIZ1 \\
\hline & Steroid metabolism & 0.85 & $A M R 1 C 2, C Y P 7 B 1, H D L B P$ \\
\hline & Synaptosome & 0.80 & $C Y F 1 P 2, D O C 2 A, S N P H$ \\
\hline \multicolumn{4}{|c|}{ Infant methylation loss } \\
\hline \multirow{4}{*}{$\begin{array}{l}\text { At } 4-6 \text { weeks } \\
\text { of life }\end{array}$} & Regulation of apoptosis & 1.61 & GIMAP1, MCF2 L, SMAD3, DLC1, DBH \\
\hline & $\begin{array}{l}\text { Antigen processing/presentation, } \\
\text { MHC class } 1\end{array}$ & 1.56 & $H L A-A, H L A-H, T A P 2$ \\
\hline & $\begin{array}{l}\text { Regulation of Rho signal } \\
\text { transduction }\end{array}$ & 1.03 & $M C F 2 L, A R H G E F 10, D L C 1, N G E F$ \\
\hline & Metabolic processes & 0.99 & COQ3, NMNAT3, PDHB \\
\hline
\end{tabular}

MHC, major histocompatibility complex. 
neurologic function also represents a key pathway for further exploration.

During lactation, maternal demands involve return to physiologic pre-pregnancy state and support of infant nutritional demands. Sufficiency in maternal vitamin D among the intervention group during lactation was associated with significant maternal leukocyte methylation gain in genes regulating cadherin signaling, adhesion complexes involved in establishment and maintenance of neural synaptogenesis and plasticity, and vascular/endothelial development. Methylation gain and loss in ion/calcium binding and hormone stimulus response align with expected associations with bone health. ${ }^{37}$ Similar to genes associated with methylation loss in pregnancy, genes in pathways regulating immune function also showed a loss of methylation during lactation among women in the intervention group. Methylation loss in transcriptional regulation among lactating women with sufficient vitamin D status also highlights the central role of methylation in gene transcription and potentially gene expression.

Emerging evidence also supports the role of DNA methylation during vulnerable periods of growth and development in driving tissue-specific gene expression, generating an infant phenotype designed to match the predicted environment. ${ }^{38}$ Current findings demonstrate inconsistent results in studies of the epigenetic effect of vitamin D targeting growthspecific imprinted differentially methylated regions. In a study of maternal vitamin D deficiency and DNA methylation on offspring growth, ${ }^{20}$ low birth weight and greater weight at 3 years were not regulated by DNA methylation in nine targeted growth-associated differentially methylated regions. Conversely, maternal vitamin D depletion affected DNA methylation at growth-associated imprinted loci in a mouse model in both germline and soma, providing evidence for transmission of epigenomic marks across generations. ${ }^{18}$

In this study, we implemented an epigenome-wide approach to identify differentially methylated loci among infants born to mothers supplemented with 400 IU (i.e., control) versus 3,800 IU (i.e., intervention) daily of vitamin D. Among infants in our sample, all of whom were exclusively or partially breastfed (high), ${ }^{2,25}$ those born to mothers in the intervention group showed methylation gain at loci with biological implications for developmental processes, including bone, lung, palate, metabolic, and nervous system. Among infants of mothers in the intervention group, methylation loss was associated with genes known to play a role in metabolic processes and signal transduction pathways, a finding also noted in examining the leukocytes of their mothers.

Also of note is the relevance of the current findings to regulation of immune function, consistent with the associations noted among vitamin D deficiency and atopic and autoimmune disorders in childhood. Our findings vary from those of Suderman et al., ${ }^{21}$ who reported no association between maternal vitamin D status and the methylation of DNA isolated from umbilical cord blood using an epigenome-wide approach. It is notable that mean maternal $25(\mathrm{OH}) \mathrm{D}$ levels, which served as a continuously measured predictor in the Suderman study, exceeded the recommended lower limit for pregnant women by more than twofold among assessed participants. Junge et al. ${ }^{39}$ have reported a significant association between vitamin D deficiency and atopy and wheezing in children in the first 3 years of life, as well as 508 significantly differentially methylated regions associated with 483 genes in children with high compared with low 25(OH)D3 levels. Taken together, evidence supports the importance of vitamin D status during critical developmental periods for improved long-term immune health.

The strengths of this study include the double-blinded randomized controlled design with placebo control and repeated measures of maternal DNA methylation at time of birth and during lactation, coupled with infant DNA methylation after 4-6 weeks of exclusive or partial (high) breastfeeding. Using a genome-wide approach, we were able to generate data which may be used to guide follow-up studies evaluating potential mechanisms, clinical care, and long-term outcomes associated with optimal vitamin D status during pregnancy and lactation with the potential to improve maternal and infant health. Study limitations include a small sample size, although findings contribute positively to the body of literature for which there are few similar studies. While infant vitamin D supplementation was highly variable, there was not a notable difference in control versus intervention group suggesting that infant $25(\mathrm{OH}) \mathrm{D}$ levels and leukocyte DNA methylation were driven by the maternal intervention and not differences by chance in infant supplementation. We were also unable to determine sustained DNA methylation changes beyond 6 weeks after birth in mothers or infants based on study design underscoring the need for further investigation. We and others ${ }^{40}$ agree that there is a need for continued investigation to determine the effect of vitamin $\mathrm{D}$ on epigenetics that extend from preconception to birth and postnatal life to generate data on pregnancy outcomes in both mothers and infants.

This pilot study and future work in this line of research may hold important clinical implications, as there is a growing body of evidence that maternal vitamin D supplementation offers benefit beyond the promotion of bone health in the absence of documented adverse effects, including by decreasing risk for preeclampsia, low birth weight, and preterm birth. ${ }^{41}$ The influence of DNA methylation on diverse biological networks represents potentially modifiable changes that can be manipulated to improve a broad range of health outcomes. To better elucidate the pathways linking differential leukocyte DNA methylation to maternal and offspring health, future validation studies will benefit from concomitant measurement of DNA methylation and expression of associated genes. Another important topic of investigation will be the identification of serum $25(\mathrm{OH}) \mathrm{D}$ levels required to avoid aberrations in specified maternal and infant biologic systems, which was not possible in this intention-totreat pilot study. Indeed, under current definitions, many pregnant women fail to avoid deficiency, $5,6,42$ which is identified according to a serum $25(\mathrm{OH}) \mathrm{D}$ value of $<20 \mathrm{ng} / \mathrm{mL}$ on the basis of the vitamin's role in the promotion of bone health. ${ }^{2}$ As studies continue to elucidate the mechanisms by which maternal vitamin D affects the health of the mother and her infant, there may be greater opportunity to produce informed prenatal care recommendations that promote both short- and long-term health across a broad range of biological systems.

\section{Conclusions}

In conclusion, this double-blind, randomized controlled pilot study provides novel evidence that maternal vitamin $\mathrm{D}$ supplementation during human pregnancy and lactation 
alters DNA methylation in mothers and breastfed infants. Among mothers, associated gene clusters showed strongest biologic relevance for cell migration/motility and cellular membrane function at birth and cadherin signaling and immune function at postpartum. Among infants, genes showing differential methylation mapped most strongly to collagen metabolic processes and regulation of apoptosis. This study adds to a small but growing body of literature suggesting that maternal vitamin D status plays an important role in shaping maternal and infant health in the prenatal period and during lactation by influencing the epigenomic landscape. Additional work is needed to fully elucidate the short- and long-term biologic effects of vitamin D supplementation at varying doses, which could hold important implications for establishing clinical recommendations for prenatal and offspring health promotion. $5,6,42$

\section{Acknowledgments}

Association of Women's Health, Obstetric and Neonatal Nursing New Investigator Award (D.K.T.); National Institutes of Health, National Center for Research Resources (NCRR) Centers of Biomedical Research Excellence (1P20GM10436001D; J.E.O., J.L.R., C.M.A.).

\section{Disclosure Statement}

No competing financial interests exist.

\section{References}

1. Abboud M, Rybchyn MS, Rizk R, et al. Sunlight exposure is just one of the factors which influence vitamin D status. Photochem Photobiol Sci 2017;16:302-313.

2. Committeee to Review Dietary Reference Intakes for Vitamin D and Calcium, Food and Nutrition Board, Institute of Medicine. In: Dietary Reference Intakes for Adequacy: Calcium and Vitamin D, Ross AC, Taylor CL, Yaktine AL, Del Valle HB, eds. Washington, DC: National Academy of Sciences, 2011, pp. 345-402, NBK56070.

3. Holick MF, Binkley NC, Bischoff-Ferrari HA, et al. Evaluation, treatment, and prevention of vitamin D deficiency: An endocrine society clinical practice guideline. J Clin Endocrinol Metab 2011;96:1911-1930.

4. Holick MF. Vitamin D and health: Evaluation, biologic functions, and recommended dietary intake for vitamin D. Clin Rev Bone Mineral Metab 2009;7:2-19.

5. Dawodu A, Davidson B, Woo JG, et al. Sun exposure and vitamin D supplementation in relation to vitamin D status of breastfeeding mothers and infants in the global exploration of human milk study. Nutrients 2015;7:1081-1093.

6. Saraf R, Morton SM, Camargo CA, Jr, et al. Global summary of maternal and newborn vitamin D status-A systematic review. Matern Child Nutr 2016;12:647-668.

7. Lu M, Xu Y, Lv L, et al. Association between vitamin D status and the risk of gestational diabetes mellitus: A metaanalysis. Arch Gynecol Obstet 2016;293:959-966.

8. Zhou SS, Tao YH, Huang K, et al. Vitamin D and risk of preterm birth: Up-to-date meta-analysis of randomized controlled trials and observational studies. J Obstet Gynaecol Res 2017;43:247-256.

9. Hypponen E, Cavadino A, Williams D, et al. Vitamin D and pre-eclampsia: Original data, systematic review and meta-analysis. Ann Nutr Metab 2013;63:331-340.
10. Hollis JL, Crozier SR, Inskip HM, et al. Modifiable risk factors of maternal postpartum weight retention: An analysis of their combined impact and potential opportunities for prevention. Int J Obes (Lond) 2017;41:1091-1098.

11. Christensen N, Sondergaard J, Fisker N, et al. Infant respiratory tract infections or wheeze and maternal vitamin D in pregnancy: A systematic review. Pediatr Infect Dis J 2017;36:384-391.

12. Sorensen IM, Joner G, Jenum PA, et al. Maternal serum levels of 25-hydroxy-vitamin D during pregnancy and risk of type 1 diabetes in the offspring. Diabetes 2012;61:175-178.

13. Wei Z, Zhang J, Yu X. Maternal vitamin D status and childhood asthma, wheeze, and eczema: A systematic review and meta-analysis. Pediatr Allergy Immunol 2016;27: 612-619.

14. Thiele DK, Ralph J, El-Masri M, et al. Vitamin D3 supplementation during pregnancy and lactation improves vitamin D status of the mother-infant dyad. J Obstet Gynecol Neonatal Nurs 2017;46:135-147.

15. Wright ML, Ralph JL, Ohm JE, et al. DNA methylation in complex disease: Applications in nursing research, practice, and policy. Nurs Outlook 2013;61:235-241.e4.

16. Hubner U, Geisel J, Kirsch SH, et al. Effect of 1 year B and D vitamin supplementation on LINE-1 repetitive element methylation in older subjects. Clin Chem Lab Med 2013;51: 649-655.

17. Amundson LA, Hernandez LL, Laporta J, et al. Maternal dietary vitamin D carry-over alters offspring growth, skeletal mineralisation and tissue mRNA expressions of genes related to vitamin $\mathrm{D}$, calcium and phosphorus homoeostasis in swine. Br J Nutr 2016;116:774-787.

18. Xue J, Schoenrock SA, Valdar W, et al. Maternal vitamin D depletion alters DNA methylation at imprinted loci in multiple generations. Clin Epigenetics 2016;8:107.

19. Meems LM, Mahmud H, Buikema H, et al. Parental vitamin D deficiency during pregnancy is associated with increased blood pressure in offspring via Panx1 hypermethylation. Am J Physiol Heart Circ Physiol 2016;311: H1459-H1469.

20. Neelon SEB, White AJ, Vidal AC, et al. Maternal vitamin $\mathrm{D}$, DNA methylation at imprint regulatory regions, and offspring weight at birth, 1 year, and 3 years. Int $J$ Obes 2017. PMID:28676681, PMCID: PMC5756131.

21. Suderman M, Stene LC, Bohlin J, et al. 25-hydroxyvitamin $\mathrm{D}$ in pregnancy and genome wide cord blood DNA methylation in two pregnancy cohorts (MoBa and ALSPAC). $J$ Steroid Biochem Mol Biol 2016;159:102-109.

22. Anderson CM, Ralph JL, Johnson L, et al. First trimester vitamin D status and placental epigenomics in preeclampsia among northern plains primiparas. Life Sci 2015;129:10-15.

23. Suitor CJ, Gardner J, Willett WC. A comparison of food frequency and diet recall methods in studies of nutrient intake of low-income pregnant women. J Am Diet Assoc 1989;89:1786-1794.

24. World Health Organization. Exclusive Breastfeeding for Optimal Growth, Development and Health of Infants. Updated 2017. www.who.int/elena/titles/exclusive_breastfeeding/ en Published December 18, 2017. (accessed December 2, 2017).

25. Labbok M, Krasovec K. Toward consistency in breastfeeding definitions. Stud Fam Plann 1990;21:226-230.

26. Anderson CM, Ralph JL, Wright ML, et al. DNA methylation as a biomarker for preeclampsia. Biol Res Nurs 2014; 16:409-420. 
27. Ohm JE, Mali P, Van Neste L, et al. Cancer-related epigenome changes associated with reprogramming to induced pluripotent stem cells. Cancer Res 2010;70: 7662-7673.

28. Bibikova M, Barnes B, Tsan C, et al. High density DNA methylation array with single $\mathrm{CpG}$ site resolution. Genomics 2011;98:288-295.

29. Dedeurwaerder S, Defrance M, Calonne E, et al. Evaluation of the infinium methylation $450 \mathrm{~K}$ technology. Epigenomics 2011;3:771-784.

30. Sandoval J, Heyn H, Moran S, et al. Validation of a DNA methylation microarray for $450,000 \mathrm{CpG}$ sites in the human genome. Epigenetics 2011;6:692-702.

31. Huang da W, Sherman BT, Lempicki RA. Bioinformatics enrichment tools: Paths toward the comprehensive functional analysis of large gene lists. Nucleic Acids Res 2009; 37:1-13.

32. Huang da W, Sherman BT, Lempicki RA. Systematic and integrative analysis of large gene lists using DAVID bioinformatics resources. Nat Protoc 2009;4:44-57.

33. Zhu H, Bhagatwala J, Huang Y, et al. Race/ethnicityspecific association of vitamin D and global DNA methylation: Cross-sectional and interventional findings. PLoS One 2016;11:e0152849.

34. Novakovic B, Sibson M, Ng HK, et al. Placenta-specific methylation of the vitamin D 24-hydroxylase gene: Implications for feedback autoregulation of active vitamin D levels at the fetomaternal interface. J Biol Chem 2009;284: 14838-14848.

35. Schulz EV, Cruze L, Wei W, et al. Maternal vitamin D sufficiency and reduced placental gene expression in angiogenic biomarkers related to comorbidities of pregnancy. J Steroid Biochem Mol Biol 2017;173:273-279.
36. Darling AL, Rayman MP, Steer CD, et al. Association between maternal vitamin D status in pregnancy and neurodevelopmental outcomes in childhood: Results from the avon longitudinal study of parents and children (ALSPAC). Br J Nutr 2017;117:1682-1692.

37. Kovacs CS. Maternal mineral and bone metabolism during pregnancy, lactation, and post-weaning recovery. Physiol Rev 2016;96:449-547.

38. Godfrey KM, Costello PM, Lillycrop KA. Development, epigenetics and metabolic programming. Nestle Nutr Inst Workshop Ser 2016;85:71-80.

39. Junge KM, Bauer T, Geissler S, et al. Increased vitamin D levels at birth and in early infancy increase offspring allergy risk-evidence for involvement of epigenetic mechanisms. J Allergy Clin Immunol 2016;137:610-613.

40. Hollis BW, Wagner CL. New insights into the vitamin D requirements during pregnancy. Bone Res 2017;5:17030.

41. De-Regil LM, Palacios C, Lombardo LK, et al. Vitamin D supplementation for women during pregnancy. Cochrane Database Syst Rev 2016;CD008873.

42. Salameh K, Al-Janahi NS, Reedy AM, et al. Prevalence and risk factors for low vitamin D status among breastfeeding mother-infant dyads in an environment with abundant sunshine. Int J Womens Health 2016;8:529-535.

Address correspondence to: Cindy M. Anderson, PhD, CRNP, ANEF, FNAP, FAHA Center for Women, Children and Youth The Ohio State University College of Nursing 1585 Neil Avenue Columbus, $\mathrm{OH} 43215$

E-mail: anderson.2675@osu.edu 